\title{
Numerical investigation on the improvement of carbon conversion in dual circulating fluidized bed reactor for chemical looping combustion of coal
}

Xi Chen, Jinchen Ma, Xin Tian, Zuwei Xu, Haibo Zhao*

State Key Laboratory of Coal Combustion, School of Energy and Power Engineering, Huazhong University of Science and Technology, Wuhan 430074, China

\section{Corresponding authors}

*Haibo Zhao, Dr Eng.

Phone: +86 278754 4779-8208, Fax: +86 278754 5526, E-mail: hzhao@mail.hust.edu.cn

ORCID: 0000-0002-2693-4499 


\section{CPFD model}

The governing equations of hydrodynamics of solid and gas phase are listed as below (Eq. 1-Eq. 10), as summarized from the work of Snider et al. ${ }^{1}$. And the Gidaspow model was chosen as the drag model in the simulation.

Mass conservation,

$\frac{\partial}{\partial t}(\alpha \rho)_{\mathrm{g}}+\nabla \cdot(\alpha \rho \boldsymbol{u})_{\mathrm{g}}=S_{\mathrm{gs}}$

Species mass conversion,

$\frac{\partial}{\partial t}\left(\rho \alpha Y_{i}\right)_{\mathrm{g}}+\nabla\left(\rho \alpha \boldsymbol{u} Y_{i}\right)_{\mathrm{g}}=-\nabla \alpha_{\mathrm{g}} \boldsymbol{J}_{\mathrm{g}, i}+\alpha_{\mathrm{g}} S_{\mathrm{g} s, i}$

Momentum conservation,

$$
\begin{aligned}
& \frac{\partial}{\partial t}(\alpha \rho \boldsymbol{u})_{\mathrm{g}}+\nabla \cdot\left(\alpha \rho \boldsymbol{u}^{2}\right)_{\mathrm{g}}=-\alpha_{\mathrm{g}} \nabla p+\alpha_{\mathrm{g}} \rho_{\mathrm{g}} \boldsymbol{g}+\nabla \cdot \boldsymbol{\tau}_{\mathrm{g}}-\beta_{\mathrm{DPM}}\left(\boldsymbol{u}_{\mathrm{g}}-\boldsymbol{u}_{\mathrm{s}}\right)+S_{\mathrm{gs}} \boldsymbol{u}_{\mathrm{g}} \\
& \beta_{D P M}=\left\{\begin{array}{lc}
150 \frac{\left(1-\alpha_{\mathrm{g}}\right)^{2} \mu_{\mathrm{g}}}{\alpha_{\mathrm{g}} d_{\mathrm{s}}^{2}}+1.75 \frac{\rho_{\mathrm{g}} \alpha_{\mathrm{s}}\left|\boldsymbol{u}_{\mathrm{g}}-\boldsymbol{u}_{\mathrm{s}}\right|}{d_{\mathrm{s}}} & \left(\alpha_{\mathrm{g}} \leq 0.8\right) \\
\frac{3}{4} C_{\mathrm{d}} \frac{\alpha_{\mathrm{g}}^{-1.65} \alpha_{\mathrm{s}} \rho_{\mathrm{g}}\left|\boldsymbol{u}_{\mathrm{g}}-\boldsymbol{u}_{\mathrm{s}}\right|}{d_{\mathrm{s}}} & \left(\alpha_{\mathrm{g}}>0.8\right)
\end{array}\right. \\
& C_{\mathrm{d}}=\left\{\begin{array}{lc}
\frac{24}{\alpha_{\mathrm{g}} \operatorname{Re}}\left[1+0.15\left(\alpha_{\mathrm{g}} \operatorname{Re}\right)^{0.687}\right] & (\operatorname{Re} \leq 1000) \\
0.44 & (\operatorname{Re}>1000)
\end{array}\right. \\
& \operatorname{Re}=\frac{\left|\boldsymbol{u}_{\mathrm{g}}-\boldsymbol{u}_{\mathrm{s}}\right| \rho_{\mathrm{g}} d_{\mathrm{s}}}{\mu_{\mathrm{g}}}
\end{aligned}
$$

Energy equation,

$$
\frac{\partial}{\partial t}(\alpha \rho H)_{\mathrm{g}}+\nabla(\alpha \rho \boldsymbol{u} H)_{\mathrm{g}}=\alpha_{\mathrm{g}} \nabla\left(\lambda_{\mathrm{g}} \nabla T_{\mathrm{g}}\right)+Q_{\mathrm{gs}}+S_{\mathrm{gs}} H_{\mathrm{g}}
$$

Particle velocity,

$$
\begin{aligned}
& \frac{\mathrm{d} \boldsymbol{u}_{\mathrm{s}}}{\mathrm{d} t}=F_{\mathrm{D}}\left(\boldsymbol{u}-\boldsymbol{u}_{\mathrm{s}}\right)+\frac{\boldsymbol{g}\left(\rho_{\mathrm{s}}-\rho\right)}{\rho_{\mathrm{s}}}+\boldsymbol{F}-\frac{1}{\rho_{\mathrm{s}}} \nabla \cdot \boldsymbol{\tau}_{\mathrm{s}} \\
& \boldsymbol{\tau}_{\mathrm{s}}=\frac{10 P_{\mathrm{s}} \alpha_{\mathrm{s}}{ }^{\beta}}{\max \left[\alpha_{\mathrm{cs}}-\alpha_{\mathrm{s}}, \varepsilon\left(1-\alpha_{\mathrm{s}}\right)\right]}
\end{aligned}
$$

Particle temperature,

$$
m_{\mathrm{s}} c_{\mathrm{s}} \frac{\mathrm{d} T_{\mathrm{s}}}{\mathrm{d} t}=h A_{\mathrm{s}}\left(T_{\infty}-T_{\mathrm{s}}\right)-f_{\mathrm{h}} \frac{\mathrm{d} m_{\mathrm{s}}}{\mathrm{d} t} H_{\text {reac }}
$$

The heat transfer coefficient between gas phase and solid phase is taken into consideration in this work, as well as the heat transfer between gas and reactor wall. The heat transfer coefficient between gas and wall can be defined as Eq. $11^{2}$, where $h_{1}, h_{\mathrm{d}}$ and $f_{\mathrm{d}}$ is heat transfer coefficient of lean phase, heat transfer coefficient of particle phase, and time-averaged ratio of wall surface covered by particle phase, respectively. And the heat transfer coefficient between gas and particle can be described by Eq. $17^{3}$. 


$$
\begin{aligned}
& h=h_{1}+f_{\mathrm{d}} h_{\mathrm{d}} \\
& \frac{h_{\mathrm{1}} L}{k_{\mathrm{f}}}=\mathrm{Nu}_{1}=0.46 \operatorname{Re}_{1}^{0.5} \mathrm{Pr}^{0.33}+3.66 \\
& \operatorname{Re}_{1}=\frac{\rho_{\mathrm{f}} u_{\mathrm{f}} L}{\mu_{\mathrm{f}}} \\
& \operatorname{Pr}=\frac{\mu_{\mathrm{f}} c_{\mathrm{p}, \mathrm{f}}}{k_{\mathrm{f}}} \\
& \frac{h_{\mathrm{d}} d_{\mathrm{p}}}{k_{\mathrm{f}}}=\mathrm{Nu}_{\mathrm{p}}=0.525 \operatorname{Re}_{1}^{0.75} \\
& f_{\mathrm{d}}=1-\mathrm{e}^{-10} \frac{\theta_{\mathrm{p}}}{\theta_{\mathrm{cp}}} \\
& \frac{h_{\mathrm{p}} d_{\mathrm{p}}}{k_{\mathrm{f}}}=\mathrm{Nu}_{\mathrm{p}}=0.37 \mathrm{Re}_{\mathrm{p}}^{0.6} \mathrm{Pr}^{0.33}+0.1 \\
& \operatorname{Re}_{\mathrm{p}}=\frac{\rho_{\mathrm{f}}\left|u_{\mathrm{f}}-u_{\mathrm{p}}\right| d_{\mathrm{p}}}{\mu_{\mathrm{f}}}
\end{aligned}
$$

The reactions and reactive kinetics involved in this work are summarized as below. The reactions include the coal pyrolysis (R1) ${ }^{4,5}$, char gasification with $\mathrm{CO}_{2} / \mathrm{H}_{2} \mathrm{O}(\mathrm{R} 2, \mathrm{R} 3)^{1,6}$, heterogeneous reactions between the $\mathrm{OC}$ and fuel gases $(\mathrm{R} 4, \mathrm{R} 5, \mathrm{R} 6)^{4,7,8}$, the oxidation of $\mathrm{OC}(\mathrm{R} 7)^{4,8}$, residual char combustion (R8) ${ }^{9}$ and water-gas shift reaction (R9) ${ }^{1,10,11 .}$

Coal $+0.001748 \mathrm{H}_{2} \mathrm{O} \rightarrow 0.4846 \mathrm{Char}+0.09427 \mathrm{CO}+0.03989 \mathrm{CO}_{2}+0.02236 \mathrm{CH}_{4}+0.01723 \mathrm{H}_{2}+0.3434 \mathrm{Ash}$

$r_{\text {pyroly }}=\left(Y_{\mathrm{a}} k_{\mathrm{a}}+Y_{\mathrm{b}} k_{\mathrm{b}}\right) C_{\text {coal }}, \quad k_{\mathrm{i}}=A_{\mathrm{i}} \exp \left(-E_{\mathrm{i}} / R T_{\mathrm{s}}\right), \quad A_{\mathrm{a}}=2 \times 10^{5} \mathrm{~s}^{-1}$,

$A_{\mathrm{b}}=1.3 \times 10^{7} \mathrm{~s}^{-1}, \quad E_{\mathrm{a}}=104.6 \mathrm{~kJ} \cdot \mathrm{mol}^{-1}, \quad E_{\mathrm{b}}=167.4 \mathrm{~kJ} \cdot \mathrm{mol}^{-1}, \quad Y_{\mathrm{a}}=0.3, \quad Y_{\mathrm{b}}=1.0$

Char $+\mathrm{CO}_{2} \leftrightarrow 2 \mathrm{CO}$

$r_{2}=1.272 m_{s} T \exp (-22645 / T)\left[\mathrm{CO}_{2}\right]-1.044 \times 10^{-4} m_{s} T^{2} \exp (-2263 / T-20.92)[\mathrm{CO}]^{2}$

Char $+\mathrm{H}_{2} \mathrm{O} \leftrightarrow \mathrm{CO}+\mathrm{H}_{2}$

$r_{2}=1.272 m_{s} T \exp (-22645 / T)\left[\mathrm{H}_{2} \mathrm{O}\right]-1.044 \times 10^{-4} m_{s} T^{2} \exp (-6319 / T-17.29)\left[\mathrm{H}_{2}\right][\mathrm{CO}]$

$m_{\mathrm{CH}_{4}}^{\mathrm{g}}=\frac{k_{\mathrm{CH}_{4}} R_{0}}{2 M W_{\mathrm{O}_{2}}} \rho_{\mathrm{s}} \varepsilon_{\mathrm{s}}\left(Y_{\mathrm{Fe}_{2} \mathrm{O}_{3}}+Y_{\mathrm{Fe}_{3} \mathrm{O}_{4}} \times \frac{S_{\mathrm{Fe}_{2} \mathrm{O}_{3}} M_{\mathrm{Fe}_{2} \mathrm{O}_{3}}}{S_{\mathrm{Fe}_{3} \mathrm{O}_{4}} M_{\mathrm{Fe}_{3} \mathrm{O}_{4}}}\right)(1-X)^{\frac{2}{3}} M W_{\mathrm{CH}_{4}}, k_{\mathrm{CH}_{4}}=\frac{3 b k_{0} e^{-E / R T}}{\rho_{\mathrm{m}} r_{0}} C_{\mathrm{CH}_{4}}^{n}$,

$\rho_{\mathrm{m}}=60627 \mathrm{~mol} \cdot \mathrm{m}^{-3}, \quad r_{0}=3.29 \times 10^{-7} \mathrm{~m}, \quad b=12, \quad k_{0}=8.0 \times 10^{-4}, E=49 \mathrm{~kJ} \cdot \mathrm{mol}^{-1}, \quad n=1.3$

$\mathrm{CO}+3 \mathrm{Fe}_{2} \mathrm{O}_{3} \rightarrow 2 \mathrm{Fe}_{3} \mathrm{O}_{4}+\mathrm{CO}_{2}$

$\stackrel{g}{m_{\mathrm{CO}}}=\frac{2 k_{\mathrm{CO}} R_{0}}{M W_{\mathrm{O}_{2}}} \rho_{\mathrm{s}} \varepsilon_{\mathrm{s}}\left(Y_{\mathrm{Fe}_{2} \mathrm{O}_{3}}+Y_{\mathrm{Fe}_{3} \mathrm{O}_{4}} \times \frac{S_{\mathrm{Fe}_{2} \mathrm{O}_{3}} M_{\mathrm{Fe}_{2} \mathrm{O}_{3}}}{S_{\mathrm{Fe}_{3} \mathrm{O}_{4}} M_{\mathrm{Fe}_{3} \mathrm{O}_{4}}}\right)(1-X)^{\frac{2}{3}} M W_{\mathrm{CO}}, \quad k_{\mathrm{CO}}=\frac{3 b k_{0} e^{-E / R T}}{\rho_{\mathrm{m}} r_{0}} C_{\mathrm{CO}}^{\mathrm{n}}$,

$\rho_{\mathrm{m}}=60627 \mathrm{~mol} \cdot \mathrm{m}^{-3}, \quad r_{0}=3.29 \times 10^{-7} \mathrm{~m}, \quad b=3, \quad k_{0}=6.2 \times 10^{-4}, \quad E=20 \mathrm{~kJ} \cdot \mathrm{mol}^{-1}, \quad n=1.0$

$\mathrm{H}_{2}+3 \mathrm{Fe}_{2} \mathrm{O}_{3} \rightarrow 2 \mathrm{Fe}_{3} \mathrm{O}_{4}+\mathrm{H}_{2} \mathrm{O}$

$m_{\mathrm{H}_{2}}^{\stackrel{g}{2}}=\frac{2 k_{\mathrm{H}_{2}} R_{0}}{M W_{\mathrm{O}_{2}}} \rho_{\mathrm{s}} \varepsilon_{\mathrm{s}}\left(Y_{\mathrm{Fe}_{2} \mathrm{O}_{3}}+Y_{\mathrm{Fe}_{3} \mathrm{O}_{4}} \times \frac{S_{\mathrm{Fe}_{2} \mathrm{O}_{3}} M_{\mathrm{Fe}_{2} \mathrm{O}_{3}}}{S_{\mathrm{Fe}_{3} \mathrm{O}_{4}} M_{\mathrm{Fe}_{3} \mathrm{O}_{4}}}\right)(1-X)^{\frac{2}{3}} M W_{\mathrm{H}_{2}}, \quad k_{\mathrm{H}_{2}}=\frac{3 b k_{0} e^{-E / R T}}{\rho_{\mathrm{m}} r_{0}} C_{\mathrm{H}_{2}}^{\mathrm{n}}$,

$\rho_{\mathrm{m}}=60627 \mathrm{~mol} \cdot \mathrm{m}^{-3}, \quad r_{0}=3.29 \times 10^{-7} \mathrm{~m}, \quad b=3, \quad k_{0}=2.3 \times 10^{-3}, \quad E=24 \mathrm{~kJ} \cdot \mathrm{mol}^{-1}, \quad n=0.8$ 
$4 \mathrm{Fe}_{3} \mathrm{O}_{4}+\mathrm{O}_{2} \rightarrow 6 \mathrm{Fe}_{2} \mathrm{O}_{3}$

$m_{\mathrm{O}_{2}}^{g}=\frac{k_{\mathrm{O}_{2}} R_{0}}{M W_{\mathrm{O}_{2}}} \rho_{\mathrm{s}} \varepsilon_{\mathrm{s}}\left(Y_{\mathrm{Fe}_{2} \mathrm{O}_{3}}+Y_{\mathrm{Fe}_{3} \mathrm{O}_{4}} \times \frac{S_{\mathrm{Fe}_{2} \mathrm{O}_{3}} M_{\mathrm{Fe}_{2} \mathrm{O}_{3}}}{S_{\mathrm{Fe}_{3} \mathrm{O}_{4}} M_{\mathrm{Fe}_{3} \mathrm{O}_{4}}}\right) X^{\frac{2}{3}} M W_{\mathrm{O}_{2}}, \quad k_{\mathrm{O}_{2}}=\frac{b k_{0} e^{-E / R T}}{\rho_{\mathrm{m}} r_{0}} C_{\mathrm{O}_{2}}^{\mathrm{n}}$,

$\rho_{\mathrm{m}}=41523 \mathrm{~mol} \cdot \mathrm{m}^{-3}, \quad r_{0}=1.8 \times 10^{-7} \mathrm{~m}, \quad b=4, \quad k_{0}=3.1 \times 10^{-4}, \quad E=14 \mathrm{~kJ} \cdot \mathrm{mol}^{-1}, \quad n=1.0$

Char $+\mathrm{O}_{2} \rightarrow \mathrm{CO}_{2}$

R8

$r_{8}=1.018 \times 10^{10} A_{s} T \exp (-10500 / T)\left[\mathrm{O}_{2}\right]$

$\mathrm{CO}+\mathrm{H}_{2} \mathrm{O} \leftrightarrow \mathrm{CO}_{2}+\mathrm{H}_{2}$

R9

$r_{9}=7.68 \times 10^{10} \exp (-36640 / \mathrm{T})[\mathrm{CO}]^{0.5}\left[\mathrm{H}_{2} \mathrm{O}\right]-6.4 \times 10^{9} \exp (-39260 / \mathrm{T})\left[\mathrm{H}_{2}\right]^{0.5}\left[\mathrm{CO}_{2}\right]$ 


\section{Model/simulation validation}

Before detailed simulation, the CPFD model is validated by comparing the data of fuel reactor (FR) flue gas concentrations and pressure distributions in the axis of FR obtained in simulation and experiment. Fig 1 shows the FR exhaust components under different operational conditions, i.e., mainly for the purpose of validating the chemical kinetics model involved in the simulation. With the change of superficial velocity of $\mathrm{FR}\left(U_{\mathrm{FR}}\right)$, temperature (T) and gasification agent concentration $\left(\mathrm{H}_{2} \mathrm{O}\right.$ ratio), it can be found that the mole fractions of $\mathrm{CO}_{2}, \mathrm{CO}, \mathrm{CH}_{4}, \mathrm{H}_{2}$ (without $\mathrm{N}_{2}$ and steam) in simulations meet the experimental measurements very well with slight difference. Moreover, the chemical reactions in the FR are also influenced by the hydrodynamics factors, so the good agreement in flue gas concentrations in Fig 1 can also validate the hydrodynamics model to some extent. Furthermore, Fig 2 shows the FR pressure distribution along the height, i.e., mainly for the purpose of validating the hydrodynamics model involved in the CPFD simulation. With the change of $U_{\mathrm{FR}}$, the average pressure data at the specified pressure detecting points monitored in experiment meet the simulation profile well. Eventually we concluded that the accuracy of model adopted in this work is satisfactory.
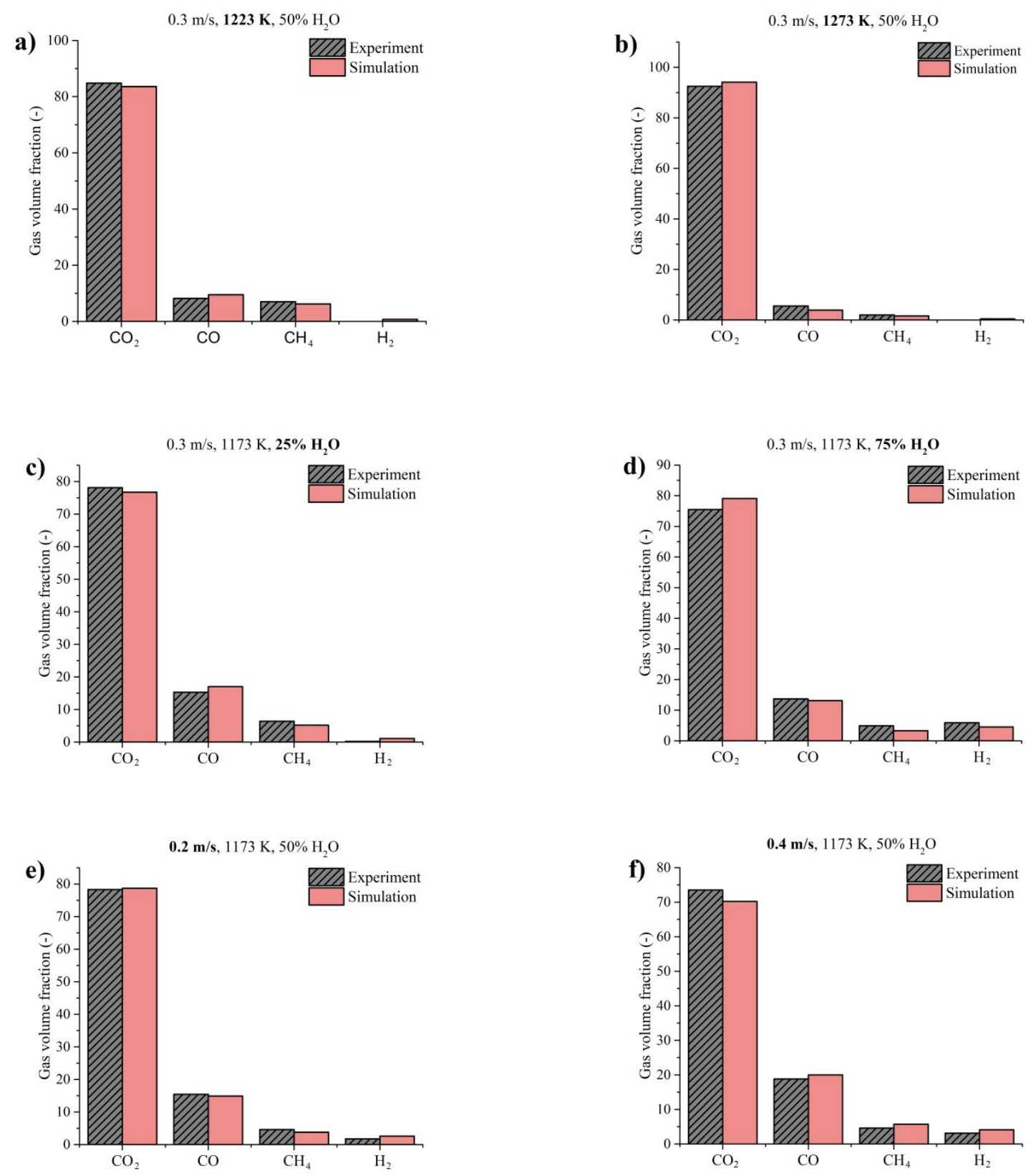
Fig 1. The fuel reactor exhaust components: experimental measurements vs. simulation results. (Case a\&b: changing the FR temperature, $T$; Case $\mathrm{c} \& \mathrm{~d}$ : changing the gasification concentration in the fluidization gas of the FR, $C_{\mathrm{H} 2 \mathrm{O}}$; Case e\&f, changing the superficial velocity of the FR, $\left.U_{\mathrm{FR}}\right)$

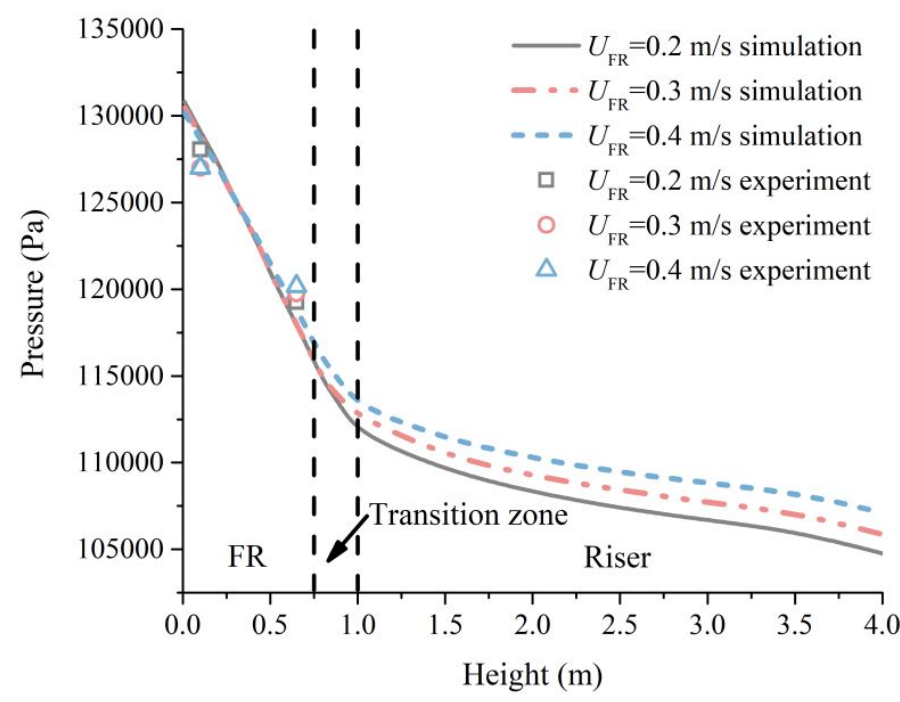

Fig 2. The pressure profile in FR: experimental measurements vs. simulation results. 


\section{Nomenclature}

\begin{tabular}{|c|c|c|c|}
\hline$A$ & Surface area $\left(\mathrm{m}^{2}\right)$ & $r$ & Reaction rate $\left(\mathrm{mol} / \mathrm{m}^{3} \mathrm{~s}\right)$ \\
\hline$A_{i}$ & Pre-exponential factor $(1 / \mathrm{s})$ & $R$ & Constant of the ideal gases $(\mathrm{J} / \mathrm{mol} \mathrm{K})$ \\
\hline$c$ & Specific heat $(\mathrm{J} / \mathrm{kgK})$ & $\operatorname{Re}$ & Reynolds number \\
\hline$C_{d}$ & Drag coefficient & $S$ & Mass source (kg) \\
\hline$C_{\text {coal }}$ & Concentration of coal $\left(\mathrm{kg} / \mathrm{m}^{3}\right)$ & $T$ & Temperature (K) \\
\hline$d$ & Diameter (m) & $u$ & Velocity vector $(\mathrm{m} / \mathrm{s})$ \\
\hline E & Activation energy $(\mathrm{kJ} / \mathrm{mol})$ & $X$ & Conversion rate \\
\hline$f_{h}$ & Heat transfer ratio & $Y$ & Mass fraction \\
\hline $\boldsymbol{F}$ & Force source $(\mathrm{N})$ & {$[\cdot]$} & Concentration $\left(\mathrm{mol} / \mathrm{m}^{3}\right)$ \\
\hline$F_{\mathrm{D}}$ & Particle drag coefficient & Greek lette & \\
\hline$g$ & Gravity $\left(\mathrm{m} / \mathrm{s}^{2}\right)$ & $\alpha$ & Volume fraction \\
\hline$h$ & Heat transfer coefficient $\left(\mathrm{J} / \mathrm{m}^{2} \mathrm{~K}\right)$ & $\varepsilon$ & $\begin{array}{l}\text { Dissipation rate of turbulent kinetic } \\
\text { energy }\left(\mathrm{m}^{-2} \mathrm{~s}^{-3}\right)\end{array}$ \\
\hline$H$ & Enthalpy (J) & $\lambda$ & Thermal conductivity $\left(\mathrm{W} / \mathrm{m}^{2} \mathrm{~K}\right)$ \\
\hline$H_{\text {reac }}$ & Enthalpy changes $(\mathrm{J} / \mathrm{kg})$ & $\rho$ & Density $\left(\mathrm{kg} / \mathrm{m}^{3}\right)$ \\
\hline$J$ & diffusion flux $\left(\mathrm{kg} / \mathrm{m}^{2}\right)$ & $\tau$ & Stress-strain tensor $(\mathrm{Pa})$ \\
\hline$k$ & $\begin{array}{l}\text { chemical reaction rate constant }\left(\mathrm{mol}^{1-\mathrm{n}} / \mathrm{m}^{2-}\right. \\
\left.{ }^{3 \mathrm{n}} \mathrm{s}\right)\end{array}$ & $\mu$ & Viscosity $(\mathrm{kg} / \mathrm{m} \mathrm{s})$ \\
\hline$m$ & Particle mass (kg) & $\begin{array}{l}\text { Subscrip } \\
\text { ts }\end{array}$ & \\
\hline$M W$ & Molecular weight (kg/mol) & $\mathrm{g}$ & Gas phase \\
\hline$n$ & Reactor order & gs & From particle phase to gas phase \\
\hline$p$ & Pressure $(\mathrm{Pa})$ & $i$ & Specie index \\
\hline$Q$ & Heat of reaction $(\mathrm{J})$ & $\mathrm{s}$ & Particle phase \\
\hline
\end{tabular}




\section{References}

(1) Snider, D. M.; Clark, S. M.; O'Rourke, P.J. Eulerian-Lagrangian method for three-dimensional thermal reacting flow with application to coal gasifiers. Chem. Eng. Sci. 2011, 66, 1285-1295.

(2) Yang, W. C. Handbook of fluidization and fluid-particle system; Marcell Dekker Inc.: New York, 2003, 262267.

(3) Kunii, D.; Levenspiel, O. Fluidization engineering, 2nd edition; Butterworth-Heinemann: Newton, 1991, 268271.

(4) Su, M.; Zhao, H.; Ma, J. Computational fluid dynamics simulation for chemical looping combustion of coal in a dual circulation fluidized bed. Energ. Convers. Manage. 2015, 105, 1-12.

(5) Wang, X.; Jin, B.; Zhang, Y.; Zhang, Y.; Liu, X. Three dimensional modeling of a coal-fired chemical looping combustion process in the circulating fluidized bed fuel reactor. Energ. Fuel. 2013, 27, 2173-2184.

(6) Syamlal, M.; Bissett, L.A. METC Gasifier Advanced Simulation (MGAS) Model. DOE/METC-92/4108, DE92 001111, 1992.

(7) Mahalatkar, K.; Kuhlman, J.; Huckaby, E. D. CFD simulation of a chemical-looping fuel reactor utilizing solid fuel. Chem. Eng. Sci. 2011, 66, 3617-3627.

(8) Abad, A.; Adanez, J.; Garcia-Labiano, F.; De Diego, L. F.; Gayán, P.; Celaya, J. Mapping of the range of operational conditions for $\mathrm{Cu}-, \mathrm{Fe}-$, and Ni-based oxygen carriers in chemical-looping combustion. Chem. Eng. Sci. 2007, 62, 533-549.

(9) Xie, J.; Zhong, W.; Shao, Y.; Liu, Q.; Liu, L.; Liu, G. Simulation of combustion of municipal solid waste and coal in an industrial-scale circulating fluidized bed boiler. Energ. Fuels 2017, 31(12), 14248-14261.

(10) Bustamante, F.; Enick, R. M.; Cugini, A. V.; Killmeyer, R. P.; Howard, B. H.; Rothenberger, K. S.; Ciocco, M. V.; Morreale, B. D.; Chattopadhyay, S.; Shi, S. High-temperature kinetics of the homogeneous reverse water-gas shift reaction. AIChE J. 2004, 50(5), 1028-1041.

(11) Bustamante, F.; Enick, R. M.; Killmeyer, R. P.; Howard, B. H.; Rothenberger, K. S.; Cugini, A. V.; Morreale, B. D.; Ciocco, M. V. Uncatalyzed and wall-catalyzed forward water-gas shift reaction kinetics. AIChE J. 2005, 51(5), 1440-1454. 